\title{
Consumo de SPA y sus efectos en la flexibilidad cognitiva en adolescentes: un estudio de revisión.
}

Pérez-Palacio, Angie Carolina ${ }^{1\left(^{*}\right)}$; Cadena-Villacrés, Leidy Ámbari'; Gómez-Cardona, Yuliza'; Serna-Moreno, Isabel ${ }^{1}$ \& Vallejo-Quintero, Ángela María ${ }^{1}$

${ }^{1}$ Universidad Católica Luis Amigó, Facultad de Psicología Medellín, Colombia.

\section{RESUMEN}

En esta investigación se abordarán como categorías de interés la flexibilidad cognitiva y los efectos que tienen las sustancias psicoactivas en la población adolescente, haciendo especial relevancia en los efectos psicológicos, neuropsicológicos y sociales que puedan incidir en la flexibilidad cognitiva a causa del consumo de spa. Se entiende flexibilidad cognitiva como la capacidad del cerebro de adaptarse a diferentes situaciones y pensamientos, y a su vez encontrar la capacidad de reajuste a diferentes contextos. Por otro lado, los investigadores y expertos definen los efectos del consumo de spa como las sustancias que producen alteración en el funcionamiento físico y mental, afectando de este modo procesos cognitivos en los adolescentes. Finalmente, la adolescencia es una etapa crucial tanto en la maduración como en el desarrollo del individuo, ya que la flexibilidad cognitiva no se encuentra desarrollada por completo en dicha etapa. En términos generales un joven sin flexibilidad cognitiva le será más difícil realizar reajustes en su conducta o adaptarse a nuevos entornos, por ello son tan vulnerables en el inicio temprano de consumo de sustancias psicoactivas las cuales tienen efectos a nivel neuropsicológico, donde se encuentra implicada la flexibilidad cognitiva. Además, la influencia de los factores de riesgo en este ciclo vital y la ausencia de factores protectores hacen a un joven más propenso hacia un consumo más allá del experimental, asimismo las conductas en esta etapa generan un impacto en la vida adulta y la salud mental posterior.

Palabras clave: Adolescencia. Flexibilidad Cognitiva. Funciones Ejecutivas. Sustancias Psicoactivas.

\section{SPA consumption and its effects on cognitive flexibility in adolescents: a review study}

\section{ABSTRACT}

In this research, cognitive flexibility and the effects of psychoactive substances on the adolescent population will be addressed as categories of interest, with special emphasis on the psychological, neuropsychological and social effects that may affect cognitive flexibility due to spa consumption. Cognitive flexibility is understood as the brain's ability to adapt to different situations and thoughts, and in turn to find the capacity to

Recibido: 24/05/2021 Aceptado:01/06/2021

Correspondencia: (*) angie.perezpa@amigo.edu.co 
readjust to different contexts. On the other hand, researchers and experts define the effects of spa consumption as substances that produce alterations in physical and mental functioning, thus affecting cognitive processes in adolescents. Finally, adolescence is a crucial stage in both the maturation and development of the individual, since cognitive flexibility is not fully developed at this stage. In general terms, a young person without cognitive flexibility will find it more difficult to readjust his or her behavior or adapt to new environments, which is why they are so vulnerable to the early onset of psychoactive substance use, which has effects at the neuropsychological level, where cognitive flexibility is involved. In addition, the influence of risk factors in this life cycle and the absence of protective factors make a young person more prone to consumption beyond the experimental stage, also behaviors at this stage have an impact on adulthood and later mental health.

Keywords: Adolescence. Cognitive flexibility. Executive functions. Psychoactive substances.

\section{INTRODUCCIÓN}

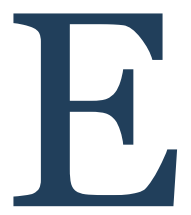

I presente trabajo pretende identificar los efectos entre el consumo de SPA y la flexibilidad cognitiva en la población adolescente, planteando los siguientes objetivos específicos: Identificar las bases neuroanatómicas implicadas en el consumo de sustancias psicoactivas y sus efectos directos en la flexibilidad cognitiva en adolescentes y a su vez describir el impacto que genera el consumo de SPA en los adolescentes logrando así determinar los factores de riesgo asociados al consumo de sustancias psicoactivas en la flexibilidad cognitiva en los adolescentes.

Esta investigación permite tener un acercamiento ante la problemática de salud pública que afecta en el presente a los adolescentes por el consumo de SPA, de tal manera que modifica la forma en que los jóvenes sienten, piensan y se comportan, y cómo esta problemática puede generar un impacto en diferentes momentos de la vida del consumidor, principalmente en la adolescencia. Así mismo es importante mencionar que este fenómeno en la actualidad va en aumento progresivo en esta población, es por tal razón que surge el interés por indagar su relación con la flexibilidad cognitiva teniendo en cuenta según Salcedo, Ramírez y Acosta (2015), que esta etapa de la vida es de vital importancia en la maduración neurológica, puesto que todavía están en desarrollo las funciones ejecutivas vitales como la habilidad de inhibición, flexibilidad cognitiva, planeación y control del comportamiento, memoria de trabajo, conciencia metacognitiva, entre otros; por lo que son fundamentales para un correcto y adaptativo funcionamiento cognitivo en los seres humanos lo que permitirá asegurar un buen desempeño en múltiples áreas de la vida.

La prevención del uso indebido de drogas es una cuestión que perjudica directa e indirectamente a las personas implicadas, por este motivo es que se hace necesario en este trabajo brindar información a los diferentes contextos sociales; de manera que, a través de esta investigación se busca originar en los lectores un interés social y conciencia sobre los efectos de la flexibilidad cognitiva por el consumo de SPA, ya que la adolescencia es una etapa crucial en el crecimiento y desarrollo del ciclo vital del individuo, la cual va a marcar el desarrollo durante esta etapa y generar secuelas con impacto en el desarrollo adulto y la salud mental posterior. Además, se considera que un adecuado manejo de la flexibilidad cognitiva tiene efectos cruciales en el bienestar mental de los adolescentes.

Finalmente, al investigar y conocer más de cerca sobre ambos temas, permitirá ampliar los conocimientos con relación a estas dos variables y a partir del rastreo bibliográfico aportar información en el ámbito académico sobre la manera como influye el consumo de SPA en la flexibilidad cognitiva en la 
población adolescente, los factores de riesgo asociados y las diferentes bases neuroanatómicas implicadas. Teniendo en cuenta que las decisiones tanto en la salud mental como físicas que se toman en la adolescencia pueden verse reflejadas en la adultez.

\section{MARCO TEÓRICO}

\section{Flexibilidad Cognitiva y adolescentes.}

Para comprender la influencia del consumo de sustancias psicoactivas en la flexibilidad cognitiva en adolescentes, hay que enmarcarla teóricamente en la comprensión de los fenómenos que ocurren en la adolescencia, haciendo referencia en el consumo de sustancias psicoactivas y la flexibilidad cognitiva en esta etapa e integrando la influencia directa que tienen estas dos variables.

La adolescencia es definida como un período de transición entre la niñez y la adultez que estimativamente se desarrolla entre los 12 y los 20/25 años, y es en esta etapa donde el adolescente intenta apropiarse de las habilidades que le confieren para una mejor adaptación y así asumir los retos que se le presentan en su cotidianidad (Acuña, Castillo, Bechara, \& Godoy, 2013). A su vez, es considerada como el período en el cual el individuo adquiere la capacidad de conciencia frente a la toma de decisiones y atraviesa desde diferentes patrones psicológicos de la niñez a los de la vida adulta (Ferrel O, Ferrel B, Alarcón \& Delgado,2016).

La adolescencia presenta aspectos particulares en la toma de decisiones, que se relacionan con factores como: la maduración del cerebro adolescente, la percepción subjetiva de los acontecimientos, los diferentes sistemas de procesamiento cognitivo de la información, la familia en su proceso de desarrollo y la sociedad en su desenvolvimiento (Luna \& Laca, 2014). Asimismo, estos autores destacan la adolescencia por ser catalogada en ser una de las etapas más cruciales de la vida de todo ser humano, requiere de destacadas habilidades de adaptación y una adecuada flexibilidad cognitiva. Es esta etapa en donde se presenta una predisposición a la toma de riesgos, ya que no se cuenta con las suficientes bases y procesos conductuales adecuados por la experiencia emocional que cada uno ha desarrollado a lo largo de su vida, por esta razón es importante tener una adecuada flexibilidad cognitiva (Michelini, Acuña, \& Godoy, 2016).

La flexibilidad cognitiva permite planear, comprender y gestionar, todas y cada una de las metas y objetivos a corto y/o largo plazo que un adolescente trace para su vida, y por tanto, reajustar la conducta, pensamiento y opiniones para adaptarse al entorno y a las nuevas situaciones que esta etapa origina, las cuales posibilitan la generación de alternativas nuevas para solucionar problemas o mejorar el desempeño cognitivo en los adolescentes (Gómez, Vargas, Hernández \& Tamayo, 2016); entre las Funciones Ejecutivas, de ahora en adelante FE, más importantes que se desarrollan en la adolescencia se encuentran el autocontrol, la memoria de trabajo, la organización, la planeación, la solución de problemas, y la flexibilidad cognitiva (Flores, Castillo \& Jiménez, 2014) mismos que son fundamentales para la toma de decisiones; las FE son relevantes para la adaptación social, vigilancia y regulación de la conducta (Camelo, Olivares, Carballeira \& Betarcort, 2019). Asimismo, elegir la mejor decisión es producto de un adecuado rendimiento de la flexibilidad cognitiva (Restrepo, 2016).

Las FE es la capacidad que tiene nuestro cerebro para adaptar nuestra conducta y pensamientos a situaciones novedosas, cambiantes o inesperadas para cada ser humano, se caracterizan como una entidad no unitaria, sino compuesta por diferentes habilidades o capacidades (García, González, Areces, Cueli \& Rodríguez, 2014). En su mayoría las FE terminan su desarrollo individual en el transcurso de la adolescencia. Las FE reciben un efecto diferencial de la escolaridad, algunas de ellas son más sensibles que otras (Flores, Castillo \& Jiménez, 2014).

El desarrollo del control inhibitorio termina su formación en la adolescencia, entre los 15 y 19 años de edad, de igual manera, en esta etapa juega un papel importante un ambiente escolarizado para que se dé un completo desarrollo de la capacidad de la planeación secuencial, la flexibilidad cognitiva, la resolución de problemas y la memoria 
de trabajo, así como el desarrollo de la abstracción y la fluidez verbal (Tamayo, Merchán, Hernández, Ramírez \& Gallo, 2018). El adecuado o correcto ejercicio del control inhibitorio es requisito indispensable para que puedan darse las funciones ejecutivas (Maddio \& Greco, 2010).

Durante el transcurso de la adolescencia se espera que los sujetos adquieran la capacidad de reconocer las contingencias asociadas a sus elecciones y regulen su comportamiento conforme a estas, lo que sería alcanzado entre los 18 y los 22 años de edad (Stelzer, Cervigni \& Martino, 2010).

\section{Calidad de vida en adolescentes consumidores de SPA.}

La calidad de vida es un conjunto de condiciones que contribuyen al bienestar de cada adolescente y a la realización de sus potencialidades en la vida social.

La adolescencia es una etapa crucial de la vida de todo ser humano por la cual el sujeto sufre diferentes cambios tanto físicos como emocionales. Esta etapa, hoy por hoy, es la más abordada por investigadores en el campo de la psicología de las adicciones, ya que ocurren cambios significativos a nivel emocional en cuanto a la toma de decisiones (Pineda,2016). Dichas decisiones tienen que ver con la calidad de vida, término que engloba los diferentes contextos en los que se desarrolla un individuo de manera equilibrada o por lo contrario de manera desequilibrada teniendo en cuenta sus aprendizajes, cultura y vivencias (Vinaccia \& Quiceno, 2012).

A medida que el adolescente crece, las condiciones de vida en las que se desenvuelven son fundamentales debido a que corresponden al modo en que las personas desarrollan su construcción individual, por el contexto histórico, político, económico y social en el que se desarrollan. Cuando se vive en contextos de escasos recursos económicos, generados por falta de empleo que a su vez se correlaciona con escaso acceso a sistemas de salud y educación. (García \& Parada, 2018). La mejora de las relaciones familiares permite proporcionarles un incremento de las expectativas y de mejores perspectivas de futuro, entre otras (Weinberg, Rahdert, Colliver y Glantz, 1998). Para Medina, Real, Villato- ro y Natera (2013), las drogas afectan los diferentes ámbitos de la vida donde se ven perjudicados en especial el desarrollo social y económico de toda la sociedad, afectando de manera significativa las inversiones que se hacen a la salud al relacionarse con lesiones y con múltiples enfermedades infecciosas (vih, hepatitis B y C) y crónicas como: cirrosis, enfermedades cardiovasculares, cáncer y enfermedades mentales. También, tienen una influencia notable en los diferentes modos de violencia donde se ven involucrados los adolescentes con las bandas delincuenciales, la venta de drogas ilegales o por el consumo e intoxicación de las sustancias psicoactivas.(Diaz \&Torres,2006; Medina ,Natera, Borges \& Cravioto,2001) El consumo crónico de drogas produce alteraciones en los diferentes ámbitos de la vida como se mencionó anteriormente y también en los circuitos cerebrales implicados en los procesos cognitivos y funciones ejecutivas del cerebro humano ya que a su vez cuando estos procesos al mismo tiempo pueden tomar un papel relevante en el continuo consumo de sustancias (García F, García O \& Secades, 2011), además del deterioro cognitivo persistente más allá del momento de la intoxicación (Beverido, 2010).

Cuando se trata de explicar los condicionantes que conlleva el consumo de drogas, se observa una gran diversidad de elementos entre los cuales se encuentran: las condiciones económicas como precarias junto con las relaciones afectivas y el círculo social en las que está inmerso el adolescente, siendo estos factores los que ayudan a la comprensión de dicha problemática social, teniendo en cuenta su gran magnitud, paso hacer una situación que no afecta sola a la salud de unos pocos sino que se convierte en un problema a gran escala, es decir, de impacto significativo, cuyas consecuencias negativas afectan no solo a la persona, sino a la sociedad en su conjunto (Arcila et al., 2020). Respecto a lo anterior el consumo en adolescentes y jóvenes es una complicación que despierta preocupación en todos los países del mundo dadas las consecuencias que acarrea desde el punto de vista de la salud, como desde lo social, cultural, ideológico y político. La salud individual, la integración familiar, el desarrollo y la estabilidad social se ven muy afectadas y carente de debido al incremento de adolescentes que ingresan diariamente al 
mundo del consumo de sustancias adictivas (Torres, Posada, Bareño \& Berbesí, 2010).

Teniendo en cuenta que los comportamientos del individuo comienzan a estar condicionados por el consumo de sustancias psicoactivas, por lo cual su proyecto de vida empieza a girar en torno a este eje en su adolescencia, hasta llegar a un consumo crónico o adictivo en el adulto joven quien desarrolla así una gran variedad de enfermedades mentales a causa del deterioro cerebral generado por este tipo de sustancias (Yen, Wang, Chen \& Chang, 2011). En la encuesta nacional de salud mental se nombran los principales trastornos mentales $y$ describe los efectos secundarios al consumo de sustancias psicoactivas, abarcando este tipo de patologías desde la intoxicación aguda que causa alteraciones psiquiátricas a raíz de la abstinencia producida por un consumo adictivo, originando también trastornos psicóticos, síndrome amnésico, entre otros que se pueden generar por el uso de una o más sustancias psicoactivas (Minsalud, 2015).

En la actualidad la sociedad está expuesta a las drogas, hay personas que tienen mayor vulnerabilidad y tendencia a sufrir consecuencias negativas por su uso, como lo son los adolescentes, quienes pueden tener muy afectado su desarrollo personal. Teniendo en cuenta que una persona haya desarrollado a lo largo del tiempo una adicción por el consumo de sustancias se considera como un proceso difícil debido a que se está se teniendo un tipo de bienestar y una evitación por algún malestar que se le presenta, sustituyendo su angustia por la dependencia de SPA donde le proporciona de forma parcial una sensación de plenitud que se instaura en su interior un círculo vicioso y nocivo debido a que tiene la necesidad de repetir continuamente la experiencia. (Laudet, 2011).

\section{Conductas adictivas.}

Conductas como el consumo de SPA se perciben como menos perjudiciales en los jóvenes que en otros períodos evolutivos de la vida (Ballester, Gil \& Girardo, 2000). Teniendo en cuenta que son muy influenciables al momento de tomar alguna decisión en los diferentes ámbitos de su vida (Caña,
Michelini, Acuña \& Godoy, 2015). En la actualidad por las diferentes problemáticas que existen y anteriormente mencionadas tienden a verse los jóvenes implicados en problemas y a su vez desarrollando conductas antisociales, lo cual ocasiona un consumo temprano de sustancias psicoactivas (Canales, Díaz, Guidorizzi \& Arena,2012). Al estudiar el bienestar psicológico de los jóvenes y sus relaciones sociales se pueden identificar diferentes estrategias de afrontamiento como la vida deportiva y las diversiones relajantes. Con respecto a la vida deportiva, cabe destacar, siguiendo a Coleman, Hendry y Kloep (2008) que el practicar ejercicio físico ayuda con la autoestima en los jóvenes, en su estado de ánimo, ayuda a prevenir la ansiedad y la depresión y a su vez ayuda a los jóvenes a evitar que se vean involucrados en el consumo de SPA. Sin embargo, la gran mayoría de los jóvenes no practican ningún tipo de actividad y se denota un alto nivel de sedentarismo el cual aumenta conforme se avanza en edad (Castillo, Balaguer, Duda \& García, 2004). Así, Madoz y Ochoa (2012) señalan una afectación de las funciones que están implicadas en la resolución de problemas entre los adolescentes consumidores, además la OMS (2015) sustenta que un tercio de la carga total de morbilidad en adultos están asociados a enfermedades o comportamientos que tienen su comienzo en la juventud lo que vuelve a resaltar la necesidad de abordar cuáles son las repercusiones y las tendencias del consumo de SPA actuales para un conocimiento adecuado de la problemática.

\section{Neurociencias, población adolescente: actuali- dad.}

El consumo de Sustancias Psicoactivas (SPA) es considerado un problema de salud pública a nivel mundial, teniendo en cuenta el impacto significativo que representa en áreas como la economía, la salud, las relaciones sociales, familiares y en el rendimiento académico de la vida de la persona que consume (Espinosa, Hernández, Cassiani, Cubides \& Martínez, 2016). En la población adolescente se hacen evidentes comportamientos de riesgo que conlleva a efectos más catastróficos en el desarrollo del individuo, debido a que en la etapa evolutiva se caracteriza por un ajuste psicosocial en proceso de crecimiento; además de cambios físicos y emo- 
cionales que posibilitan la construcción del ser humano en aspectos como la personalidad e identidad, a través del proceso de socialización e interacción con el entorno, que expone fácilmente diversos riesgos, lo cual los hace más vulnerables al comienzo del consumo experimental (Rodríguez Ramos, Madariaga, Arrivillaga \& Galende, 2016).

Por otro lado, el consumo de SPA se relaciona de forma directa con en las alteraciones en los procesos psicológicos como lo son: la memoria, atención, e inicialmente en las funciones ejecutivas en las cuales está inmersa la flexibilidad cognitiva (Piñón et al., 2016). Las funciones ejecutivas se han visto involucradas en las tareas de seguimiento y ordenación de los procesos cognitivos superiores, en el momento de realizar diferentes actividades complejas (Miyake, Friedman, Emerson, Witzki, Howerter \& Wager, 2000).

Otros autores como Tirapu, García, Ríos y Ardila (2012): hacen referencia a las funciones ejecutivas como las destrezas que tenemos para resolver conflictos y hallar soluciones, las cuales se han asociado principalmente con el Córtex prefrontal, como lo muestran algunos de los estudios de personas con lesiones en la corteza prefrontal, donde se evidencia bajo nivel en las aplicaciones de pruebas como el Wisconsin (Longo, Kerr, Smith, 2013) y Torre de Londres (Foster, Kisley, Davis, Diede, Campbell \& Davalos, 2013) y algunas investigaciones realizadas en Colombia por Mariño, Castro y Torrado (2012) sobre las alteraciones en funciones ejecutivas en policonsumidores de sustancias psicoactivas, establecen que en las áreas frontales, las zonas con mayor nivel de alteración cognitiva como la corteza motora premotora encargada de la capacidad de planear, la corteza fronto-medial, responsable de la atención; y la corteza prefrontal dorsolateral, responsable de acciones como la memoria, la fluidez y la flexibilidad mental; tareas importantes en el establecimiento de metas, la adecuada selección de conductas y su organización en el espacio y en el tiempo, la flexibilidad cognitiva en la monitorización de estrategias, la supervisión de las conductas en función de los estados motivacionales y afectivos, y en la toma de decisiones.
Las Funciones ejecutivas están relacionadas y ubicadas en diferentes partes de la corteza prefrontal, específicamente en la habilidad de mantener información en la memoria de trabajo, ubicada en la zona lateral de la corteza prefrontal (Narayanan, Prabhakaran, Bunge, Christoff, Fine \& Gabrieli, 2005); La flexibilidad ha sido ubicado en la zona medial de la corteza prefrontal (Crone, Wendelken, Donohue \& Bunge, 2005); la habilidad de inhibición de respuestas ha sido relacionada con la corteza orbitofrontal (Aron, Robbins \& Poldrack, 2004) y con otras estructuras ya que se trata de evitar una postura reduccionista y localizacionista. Lo que demuestra la suma importancia que tiene el desarrollo cognitivo ya que es, por consiguiente, en el desarrollo del cerebro (Casey,Tottenham, Liston \& Durstonet, 2005).

El consumo alargado y la adicción a las sustancias psicoactivas se le atribuye al detrimento de funciones neuropsicológicas, por su impacto clínico en los jóvenes adictos (Verdejo, Orozco, Sánchez, Aguilar \& Pérez, 2004).

Estos resultados, han inquietado y posibilitado que se produzca un desarrollo progresivo en la investigación neuropsicológica sobre los déficits causados por la adicción. El interés por investigar las alteraciones en las capacidades cognitivas, como las funciones ejecutivas se explica, entre otras razones, porque se ha demostrado que el abuso consumo prolongado de drogas ocasiona alteraciones en las estructuras anatómicas y fisiológicas del cerebro los cuales para Ruiz y Pérez (2014) pueden mantenerse en zonas cerebrales que involucran funciones cognitivas, especialmente la flexibilidad cognitiva.

\section{Características o elementos descriptivos del adolescente consumidor.}

El ser humano se caracteriza por ver el mundo desde la curiosidad, el experimentar nuevas sensaciones hace parte de lo que llamamos vivir, lo que puede conducir a alterar y percibir la vida desde diferentes estados de conciencia y en muchas ocasiones conllevan a evitar la realidad. Las SPA apuntan ya sea directa o indirectamente en el funcionamiento del sistema nervioso central 
(SNC) y en general todas tienen efectos adversos que podemos ver y medir (Cáceres et al., 2016). Asimismo, con base en la sustancia, frecuencia y cantidad de consumo se producen cambios a nivel social, personal, cerebral, físicos y psicológicos (Correa \& Pérez, 2013).

Las drogas impactan en los circuitos de recepción cerebrales y originan dependencia y adicción, lo que se define como trastorno por consumo de sustancias (Méndez, Romero, Cortés, Ruíz \& Prospero, 2017). Dicho trastorno se caracteriza por un consumo abusivo y continuo de drogas que se refleja en síntomas cognitivos, comportamentales y fisiológicos que indican que la persona continúa consumiendo la sustancia a pesar de los problemas significativos que provoca en el individuo. Tal como se recoge en los criterios diagnósticos del DSM-V para su diagnóstico se tienen en cuenta 11 criterios los cuales se dividen de la siguiente manera: leve ( 3 criterios) y grave (más de 6 criterios), y las diferentes sustancias se clasifican en: alcohol, cafeína, cannabis, alucinógenos (fenciclidina y otros), inhalantes, opiáceos, sedantes, hipnóticos, ansiolíticos, estimulantes (anfetaminas, cocaína y otros), tabaco y otras sustancias (American Psychiatric Association (APA), 2014).

En la actualidad se le ha dado mayor relevancia al tema del consumo de sustancias en los adolescentes, puesto que en los últimos años ha venido en aumento y en la mayoría de países del mundo ha disminuido considerablemente la edad de consumo (Moreno \& Palomar, 2017). Algunos estudios, como el de Vázquez, Muñoz, Fierro, Alfaro, Rodríguez C y Rodríguez M (2014) destacan como edad promedio de consumo de alcohol y tabaco entre los 13 y 14 años, y con respecto a las drogas ilegales entre los 15 y 16 años, así como la influencia de factores de riesgo que inciden en conductas de consumo tempranas. Por otro lado, se evidencia que, a menor edad de inicio de consumo, existe una mayor probabilidad de riesgo de adicción (Bousoño et al., 2019). Al igual que un incremento de la presencia de déficits neurocognitivos (Molero, Pérez \& Vásquez, 2017).

Diversas investigaciones, nombran como principales factores que se relacionan con el consumo de SPA: la sintomatología depresiva, la influencia social, disponibilidad de drogas en el entorno, consumo al interior de la familia, abandono escolar, factores biológicos y psicológicos, uso de sustancias como alcohol y tabaco; familias disfuncionales que carecen de apoyo para los adolescentes, desconocimiento de los efectos y consecuencias que provocan a largo plazo en la salud y las relaciones sociales, así como la alta exigencia académica que puede generar que los jóvenes consuman para aumentar el rendimiento (Tarter, Vanyukov, Kirisci, Reynolds \& Clark, 2006; Wongtongkam, Ward, Day \& Winefield, 2014; Loor, Hidalgo .Macías, García \& Scrich, 2018). Por el lado de las relaciones sociales, el adolescente se puede ver influenciado por las conductas del grupo con el que se relaciona, ya que tiende a imitar o reproducir los comportamientos que ve al interior del mismo para poder ser aceptado (Branstetter, Low \& Furman, 2011). Estos factores de riesgo son multicausales, así como el ambiente social puede incitar al consumo, un entorno familiar desestructurado, con progenitores que consumen algún tipo de sustancia psicoactiva, o son más permisivos, motivan a que los hijos adolescentes sean más propensos al consumo de drogas (Peñafiel, 2009; Bousoño et al., 2019). Con respecto a la familia es el factor más recurrente mencionado, que incide en el inicio del consumo de SPA en jóvenes; estudios como el de Baba et al. (2013) indican una prevalencia del 57\%, además sugiere que características como el sexo y la edad también son factores de riesgo que pueden desencadenar conductas de abuso de sustancias en los jóvenes.

Según el último estudio realizado por Simón et al. (2020) a diferencia de otras drogas, el alcohol al ser una sustancia legal en muchos países y presentar mayor disponibilidad, es considerada por los jóvenes como una sustancia de menor percepción de daño, por tal razón es la más utilizada y la puerta de entrada para el consumo de sustancias ilegales como el tabaco y cannabis las cuales continúan la lista de las más consumidas en los adolescentes. Igualmente, se identificaron algunos elementos protectores que evitan el consumo regular, entre ellos se destacan: un buen estado de ánimo, apoyo familiar y académico, autoconcepto positivo y se le suma la espiritualidad; estos factores pueden contrarrestar el efecto que conlleva a un abuso de 
SPA y posibilita la creación de la resiliencia en los adolescentes frente a las conductas de consumo (Nikmanesh \& Honakzehi, 2016).

A lo que concierne con el abuso de SPA, un porcentaje más alto de jóvenes se quedan en una etapa experimental, así como es probable que otros consuman de forma regular lo que en consecuencia trae dependencia a las mismas (Simón et al., 2020).

\section{METODOLOGÍA}

Para llevar a cabo los objetivos propuestos en el presente trabajo, se realizó un rastreo bibliográfico en fuentes académicas rigurosas, con el fin de hallar diferentes estudios científicos, en los diferentes periodos, clásicos, modernos y contemporáneos, donde se dio paso a seleccionar las investigaciones que relacionaban las categorías del consumo de SPA y sus efectos en la flexibilidad cognitiva en población adolescente, adicionalmente, se indagó en las bases neuroanatómicas implicadas, el impacto que genera una variable en la otra y los factores de riesgo y protectores asociados, según los estudios realizados. Las investigaciones que formaron parte de la revisión bibliográfica fueron estudios tomados a partir del año de 2010, exceptuando un estudio del 2000 que fue necesario citar en correspondencia con las categorías de estudio. Los artículos fueron tomados de bases de datos tales como: PubMed, Dialnet, Scielo, Scopus, Redalyc, Google Académico y EBSCO. Para la estructuración y exploración del material, se hizo un listado de categorías donde se remitieron datos como: autor, año, referencia bibliográfica, país de publicación y los términos que se utilizaron para la búsqueda fueron: Funciones Ejecutivas, flexibilidad cognitiva en adolescentes, consumo SPA en adolescentes, neuropsicología de las funciones ejecutivas, SPA y factores de riesgo.

En esta revisión del consumo de SPA y la flexibilidad cognitiva, se incluyeron artículos empíricos y también de revisión. Se tomaron publicaciones a partir del desarrollo del tema de la flexibilidad cognitiva y/o funciones ejecutivas y consumo de SPA en adolescentes. Se seleccionaron estudios investigados únicamente en población adolescente. Asimismo, los artículos que no pertenecen a una fuente no confiable fueron excluidos, también, se excluye- ron aquellos artículos en donde las variables de consumo de SPA y flexibilidad cognitiva no se nombraran.

Se tuvieron en cuenta los artículos realizados con población adolescente y se revisaron, aproximadamente, por cada estudiante 20 artículos, de los cuales solo 64 formaron parte del presente estudio por cumplir con los criterios de inclusión.

\section{DISCUSIÓN}

El consumo de SPA afecta el desempeño ejecutivo y produce efectos negativos relevantes a nivel neuropsicológico especialmente en la flexibilidad cognitiva de los adolescentes. Esta teoría es coherente con los resultados del estudio de Verdejo, Orozco, Meersmans, Aguilar y Pérez (2004), donde se reitera que el consumo de SPA y la adicción a algunas drogas se encuentra estrechamente relacionada con el deterioro neuropsicológico, destacando la inherencia del desarrollo de un individuo en los diferentes entornos y la influencia de las relaciones sociales. Así mismo, Mariño, Castro y Torrado (2012) determinaron a través de estudios de caso y controles del rendimiento ejecutivo en policonsumidores de SPA, la existencia de alteraciones cognitivas y deterioro en la corteza dorsolateral que se encarga de acciones como la flexibilidad cognitiva, encontrando que las alteraciones en esta función pueden estar relacionadas con dificultades al afrontar situaciones imprevistas y la capacidad de resolución de conflictos lo que también se vincula al deterioro neuropsicológico.

En cuanto a la adolescencia es definida como una etapa de transición donde se sufren cambios biológicos, cognitivos, emocionales y sociales; en este periodo se experimentan nuevos comportamientos y experiencias, algunos de los cuales pueden representar un factor de riesgo para la salud como lo es el consumo de drogas (Gaete, 2015). Dado que en la actualidad se ha reducido considerablemente la edad de consumo, en los adolescentes la prevalencia de uso y abuso de drogas se encuentra en constante aumento representando un fenómeno social que causa preocupación, el cual genera problemas en torno a la salud mental de esta población y que repercuten a la vida adulta. De la misma manera, autores como Bousoño et al. (2019) ratifican que, a menor edad de inicio de consumo, se aumenta la 
probabilidad de riesgo de adicción y no solo eso, sino que también se intensifica la posibilidad de presentar un déficit neurocognitivo. Entendiendo que esta etapa el individuo se encuentra en pleno proceso de maduración cerebral y sus funciones ejecutivas no se encuentran desarrolladas por completo, para un joven es más complicado tomar decisiones, realizar reajustes en su conducta, adaptarse a nuevos entornos, lo cual los hace más vulnerables al inicio de consumo de sustancias (Molero, Pérez \& Vásquez, 2017).

Finalmente, es importante aclarar que el consumo de drogas no aparece de la nada en la adolescencia, sino que existen diversos factores que aumentan o disminuyen el riesgo al consumo o abuso. Autores como López y Rodríguez (2010) y a partir de investigaciones que iniciaron en los años ochenta y otras igualmente contrastadas concuerdan con que el consumo de drogas se relaciona con factores de riesgo en la familia, la comunidad, la escuela, el individuo y los pares, lo que más tarde se corrobora en investigaciones como las de Loor, Hidalgo. Macías, García y Scrich, (2018) que amplían otros factores como sintomatología depresiva, la influencia social, disponibilidad de drogas, factores biológicos y psicológicos, uso de sustancias como alcohol y tabaco; baja percepción del riesgo o desconocimiento de los efectos y consecuencias que provocan a largo plazo en la salud, alta exigencia académica, entre otros. Todos estos identificando a la familia como el principal entorno de riesgo y por el lado de los factores protectores los autores coinciden que un buen estado de ánimo, apoyo familiar y académico, un autoconcepto positivo, pueden contrarrestar el efecto que conlleva a un abuso de SPA y posibilita la creación de la resiliencia (Nikmanesh \& Honakzehi, 2016).

\section{CONCLUSIONES}

En el paso por la adolescencia se experimenta una notable inestabilidad, producto de la combinación de cambios psicológicos, biológicos y sociales, donde se encuentra la probabilidad de comenzar a explorar y experimentar, de allí que en algunas circunstancias puede aparecer el abuso de las drogas, el consumo de alcohol o de otras sustancias, que pueden dar lugar al abuso y dependencia de las mismas a nivel fisiológico, psicológico o ambas y así mismo la posibilidad de desencadenar en el adolescente que consume sustancias, una afección notable en la flexibilidad cognitiva. Por tanto, lo anteriormente mencionado, no afecta solamente al adolescente en esa etapa del desarroIlo, sino que puede tener grandes repercusiones en su vida adulta, por la cantidad de afecciones que genera en todos los ámbitos de su vida.

Según las investigaciones más recientes demuestran que con el consumo prolongado de SPA se obtienen grandes alteraciones de orden cognitivos específicamente en la atención, la memoria y las funciones ejecutivas.

Identificar las afecciones que puedan manifestar los adolescentes consumidores de SPA son de sumo valor, ya que se conocerá, en qué áreas posee mayor impacto (Académicas, familiares, social) y así conocer la ruta más accesible el tratamiento. 


\section{REFERENCIAS}

Acuña, I., Castillo, D., Bechara, A., \& Godoy, J. C. (2013). Toma de decisiones en adolescentes: rendimiento bajo diferentes condiciones de información e intoxicación alcohólica. International Journal of Psychology and Psychological Therapy, 13(2), 195-214. http://www.redalyc.org/articulo.oa?id=56027416004

American Psychiatric Association(APA). (2014). Trastornos relacionados con sustancias o trastornos adictivos. En DSM-5. Manual Diagnóstico y Estadístico de los Trastornos Mentales (5.a ed., pp. 481-590). Editorial Médica Panamericana.

Arcila Echavarría, D., Reyes Ruíz, L., Carmona Alvarado, F., Méndez Morón, S., Morales Márquez, J., Morrón Gómez, C., \& Pérez Castro, C. (2020). Factores psicosociales asociados al consumo de sustancias psicoactivas en jóvenes universitarios entre los 16 y 24 años. Ciencia, Tecnología e Innovación en salud, 4. https://doi.or$\mathrm{g} / 10.23850 / 25393871.2876$

Aron, A. R., Robbins, T. W., \& Poldrack, R. A. (2004). Inhibition and the right inferior frontal cortex. Trends in cognitive sciences, 8(4), 170-177. https://doi.org/10.1016/j.tics.2004.02.010

Baba, T., Ganai, A., Qadri, S., Margoob, M., Iqbal, Q., \& Khan, Z. (2013). An epidemiological study on substance abuse among college students of north India (Kashmir valley). International Journal of Medical Science and Public Health, 2(3), 562 . htt ps: / / d o i . org / 10.5455 / i jmsph.2013.080420131

Ballester, R., Gil, M., \& Guirado, M.. (2000). Behaviours and attitudes related to alcohol consumption in adolescents between 15 and 17 years old. Annual Modification Conductual, 26, 855-895.

Bausela Herreras, E. (2014). Funciones ejecutivas: nociones del desarrollo desde una perspectiva neuropsicológica. Acción Psicológica, 11(1), 21-34. https://dx.doi.org/10.5944/ap.1.1

Becoña, E. (2000). Los adolescentes y el consumo de drogas. Papeles del Psicólogo, 77, 25-32. https://www.redalyc.org/articulo.oa?i$\mathrm{d}=77807705$
Beverido, S. (2010). Consumo de mariguana y sus efectos en la salud mental y las habilidades cognitivas necesarias para el aprendizaje. Rev Med UV, 10(2), 49-53. https://www.medigraphic.com/cgi-bin/new/resumen.cgi?IDARTICU$\mathrm{LO}=29259$

Bousoño Serrano, M., Al-Halabí, S., Burón, P., Garrido, M., Díaz-Mesa, E. M., Galván, G., García-Álvarez, L., Velasco, Á., Wasserman, C., Carli, V., Hoven, C., Sarchiapone, M., Wasserman, D., Bousoño, M., García-Portilla, M. P., Iglesias, C., Sáiz, P. A., \& Bobes, J. (2019). Factores predictores del consumo de alcohol en adolescentes: datos de un estudio prospectivo de 1 año de seguimiento. Adicciones, 31(1), 52-63. https://doi.org/10.20882/adicciones.998

Branstetter, S. A., Low, S., \& Furman, W. (2011). The Influence of Parents and Friends on Adolescent Substance Use: A Multidimensional Approach. Journal of substance use, 16(2), 150-160. $\mathrm{h} t \mathrm{t} \quad \mathrm{p}$ : / / d o i . o r g/10.3109/14659891.2010.519421

Cáceres, C. F., León, L. M. L., Torre, Ó. L., ProQuest, \& de la Torre, Ó. L. (2016). Factores Neurobiológicos y genéticos. En Cerebro y sustancias psicoactivas (3-7). Centros de Integración Juvenil.

Camelo, S. M., Olivares, T., Carballeira, M., \& Betancort, M. (2019). Funciones Ejecutivas y Ajuste Clínico en Adolescentes Colombianos Policonsumidores. Terapia psicológica, 37(2), 141-153. $\mathrm{h}$ tt $\mathrm{p}$ s : / / d o i . o r $\mathrm{g} / 10.4067 / \mathrm{s} 0718-48082019000200141$

Canales, G., Díaz, T., Guidorizzi, A., \& Arena, C. (2012). Consumo de drogas psicoactivas y factores de riesgo familiar en adolescentes. Revista CUIDARTE, 3(1), 260-269. https://doi.or$\mathrm{g} / 10.15649 /$ cuidarte.v3i1.20

Caña, M., Michelini, Y., Acuña, I., \& Godoy, J. (2015). Efectos de la impulsividad y el consumo de alcohol sobre la toma de decisiones en los adolescentes. Health and Addictions/Salud y Drogas, 15(1), 55-66. https://doi.org/10.21134/haaj.v15i1.231 
Casey, B. J., Tottenham, N., Liston, C., \& Durston, S (2005). Imaging the developing brain: what have we learned about cognitive development?. Trends in cognitive sciences, 9(3), 104-110. https://doi.org/10.1016/j.tics.2005.01.011

Castillo, I., Balaguer, I., Duda, J.L. y García Merita, M.L. (2004). Factores psicosociales asociados con la participación deportiva en la adolescencia. Revista Latinoamericana de Psicología, 36 (3), 505-515.

Coleman, J., Hendry, L. B., \& Kloep, M. (2008). Adolescencia y salud (1.a ed.). El manual moderno

Correa Muñoz, A. M., \& Pérez Gómez, A. (2013). Relación e impacto del consumo de sustancias psicoactivas sobre la salud en Colombia. Liberabit, 19(2), 153-162. http://www.scielo.org.pe/scielo.php ? script =sci_arttext\&pid=S1729-48272013000200001

Crone, E. A., Wendelken, C., Donohue, S. E., \& Bunge, S. A. (2005). Neural Evidence for Dissociable Components of Task-switching. Cerebral Cortex, 16(4), 475-486. https://doi.or$\mathrm{g} / 10.1093 /$ cercor/bhi127

Díaz, C., \& Torres, M. (2006). Complicaciones neurológicas por cocaína. Revista Adicciones, 18, 179-196. https://medes.com/publication/22898

Espinosa Soto, K., Hernández Carrillo, M., Cassiani, C. A., Cubides Munevar, Á. M., Martínez Cardona, M. C. (2016). Factores relacionados con el consumo de sustancias psicoactivas en una institución educativa de Jamundí Valle, Colombia. Revista Colombiana de Psiquiatría, 45(1), 2-7. https://doi.org/10.1016/j.rcp.2015.06.001

Ferrel Ortega, F. R., Ferrel Ballestas, L., Alarcón Baquero, A., \& Delgado Arrieta, K. (2016). El consumo de sustancias psicoactivas como indicador de deterioro de la salud mental en jóvenes escolarizados. Psychologia, 10(2), 43-54. https://doi.org/10.21500/19002386.2552
Flores, J. C., Castillo-Preciado, R. E., \& Jiménez-Miramonte, N. A. (2014). Desarrollo de funciones ejecutivas, de la niñez a la juventud. Anales de Psicología, 30(2), 463-473. https://doi.org/10.6018/analesps.30.2.155471

Foster, S. M., Kisley, M. A., Davis, H. P., Diede, N. T., Campbell, A. M., \& Davalos, D. B. (2013). Cognitive function predicts neural activity associated with pre-attentive temporal processing. Neuropsychologia, 51(2), 211-219. https://doi.org/10.1016/j.neuropsychologia.2012.09.017

Gaete, V. (2015). Desarrollo psicosocial del adolescente. Revista Chilena de Pediatría, 86(6), 436-443. $\quad$ https://doi.org/10.1016/j.rchipe.2015.07.005

García Fernández, G., García Rodríguez, O., \& Secades Villa, R. (2011). Neuropsicología Y Adicción A Drogas. Papeles del Psicólogo, 32(2), 159-165. https://www.redalyc.org/articulo.oa?i$d=77818544005$

García Fernández, T., González Castro, P., Areces, D., Cueli, M., \& Rodríguez Pérez, C. (2014). Funciones ejecutivas en niños y adolescentes: implicaciones del tipo de medidas de evaluación empleadas para su validez en contextos clínicos y educativos. Papeles del Psicólogo, 35(3), 215-223. h tt p s: / / w w w. red a I y c . org / pd f/778/77832241007.pdf

García Suárez, C., \& Parada Rico, D. (2018). “Construcción de adolescencia": una concepción histórica y social inserta en las políticas públicas. Universitas Humanística, 85(85). https://doi.or$\mathrm{g} / 10.11144 /$ javeriana.uh85.cach

Giraldo Franco, M. A. (2017) Condiciones de vida en el consumo inicial de sustancias psicoactivas en el adolescente y en el consumo adictivo del adulto joven con enfermedad mental [Tesis De Pregrado, Universidad De Ciencias Aplicadas Y Ambientales U.D.C.A,]. Repositorio Institucional -Universidad De Ciencias Aplicadas Y Ambientales U.D.C.A. 
Gómez Calvo, M. J., Vargas Mendoza, V. A., Hernández, J., \& Tamayo Lopera, D. (2016). Flexibilidad cognitiva en estudiantes de la I.E. Manuel Uribe Ángel del Municipio Envigado-Colombia. Psicoespacios, 10(17), 41. https://doi.or$\mathrm{g} / 10.25057 / 21452776.801$

Laudet A. B. (2011). The case for considering quality of life in addiction research and clinical practice. Addiction science \& clinical practice, 6(1), 44-55.

Loor Briones, W., Hidalgo Hidalgo, H., Macías Alvarado, J., García Noy, E., \& Scrich Vázquez, A. (2018). Causas de las adicciones en adolescentes y jóvenes en Ecuador. Archivo Médico Camagüey, 22(2), 130-138. http://www.revistaamc.sld.c u / i nd ex.ph p / a m c/a r ti cle / view/5578/3081(REVISAR

Mulsow, G. (2008). Desarrollo emocional: impacto en el desarrollo humano. revista Educação. ISSN: 0101-465X, 61-65.

López, S., \& Rodríguez, L. (2010). Factores de riesgo y de protección en el consumo de drogas en adolescentes y diferencias según edad y sexo. Psicothema, 2(4), 568-573. https://www.redalyc.org/articulo.oa?id=72715515007

Luna Bernal, A. C., \& Laca Arocena, F. A. V. (2014). Patrones de toma de decisiones y autoconfianza en adolescentes bachilleres. Revista de Psicología, 32(1), 39-65. https://doi.org/10.18800/psico.201401.002

Maddio, L., \& Greco, C. (2010). Flexibilidad Cognitiva para Resolver Problemas entre Pares ¿Difiere esta Capacidad en Escolares de Contextos Urbanos y Urbanomarginales? Revista Interamericana de Psicología/Interamerican Journal of Psychology, 44(1), 98-109. https://www.redalyc.org/pdf/284/28420640011.pdf

Madoz Gúrpide, A., \& Ochoa Mangado, E. (2012). Alteraciones de funciones cognitivas y ejecutivas en pacientes dependientes de cocaína: estudio de casos y controles. Revista de Neurología, 54(04), 199. https://doi.org/10.33588/rn.5404.2011174
Mariño, Nathaly, Castro, Jenny, \& Torrado, Javier. (2012). Funcionamiento ejecutivo en policonsumidores de sustancias psicoactivas. Revista de Psicología Universidad de Antioquia, 4(2), 49-64. http://pepsic.bvsalud.org/scielo.php?script=s$c \quad i \quad a \quad t$ e $x$ t \& $p$ i $d=S 2145-48922012000200004 \& \operatorname{lng}=p t \& t \operatorname{lng}=e s$

Medina, M., Natera, G., Borges, G. \& Cravioto, P. (2001). Del siglo XX al tercer milenio. Las adicciones y la salud pública: drogas, alcohol y sociedad. Salud Mental, 24(4), 3-19. https://www.redalyc.org/articulo.oa?i$d=58242402$

Medina, M., Real, T., Villatoro, J., \& Natera, G. (2013). Las drogas y la salud pública: ¿hacia dónde vamos? Salud Pública de México, 55(1), 67-73. https://doi.or$\mathrm{g} / 10.1590 / \mathrm{s} 0036-36342013000100010$

Méndez Díaz, M., Romero Torres, B. M., Cortés Morelos, J., Ruíz Contreras, A. E., \& Prospéro García, O. (2017). Neurobiología de las adicciones. Revista de la Facultad de Medicina, 60(1), 1-11. http://www.scielo.org.mx/scielo.php?pid=S0026-17422017000100006\&script=sci_artte $x t$

Michelini, Y., Acuña, I., \& Godoy, J. C. (2016). Emociones, toma de decisiones y consumo de alcohol en jóvenes universitarios. Suma Psicológica, 23(1), 42-50. https://doi.org/10.1016/j.sumpsi.2016.01.001

MinSalud. (2015). Encuesta Nacional de Salud Mental (N.o 4). Ministerio de Salud y Protección Social. https://www.minsalud.gov.co/sites/rid / L i s t s/B i b l i o t e c a D i g i tal/RIDE/DE/abc-encuesta-nacional-salud-ment al-2015.pdf

Miyake, A., Friedman, N. P., Emerson, M. J., Witzki, A. H., Howerter, A. y Wager, T. D. (2000). The unity and diversity of executive functions and their contributions to complex "frontal lobe" tasks: A latent variable analysis. Cognitive Psychology, 41, 49-100. 
Molero-Jurado, M. M., Pérez-Fuentes, M. C., Gázquez-Linares, J. J., \& Barragán-Martín, A. B. (2017). Análisis y perfiles del consumo de drogas en adolescentes: percepción del apoyo familiar y valoración de consecuencias. Atención Familiar, 24(2), 56-61. https://doi.org/10.1016/j.af.2017.02.001

Moreno Carmona, N. D., \& Palomar Lever, J. (2017). Factores familiares y psicosociales asociados al consumo de drogas en adolescentes. Interamerican Journal of Psychology, 51(2), 141-151. https://www.redalyc.org/articulo.oa?i$d=28454546001$

Narayanan, N. S., Prabhakaran, V., Bunge, S. A., Christoff, K., Fine, E. M. y Gabrieli, J. D. E. (2005). The role of the prefrontal cortex in the maintenance of verbal working memory: An event-related fMRI analysis. Neuropsychology, 19 (2), $223-232$. h tt p s : / / d o i . o r $\mathrm{g} / 10.1037 / 0894-4105.19 .2 .223$

Nikmanesh, Z., \& Honakzehi, F. (2016). Examining Perceived Social Support, Positive Affection, and Spirituality, as Resilience Factors, Among Boys of Drug-Dependent Fathers. Shiraz E-Medical Journal, 17(12), 1-7. https://doi.org/10.17795/semj42200

Organización Mundial de la Salud. (2015). Informe mundial sobre el envejecimiento y la salud. Organización Mundial de la Salud.

Peñafiel, E. (2009). Factores de riesgo y protección en el consumo de sustancias en adolescentes. Pulso, 32, 147-173.

Piñon Blanco, A., Lage López, M., Carrera Machado, I., Vázquez González, M., Cerqueiro Costa, X., Bobadilla Pérez, V., Spuch Calvar, C., \& Otero Lamas, F. (2019). Perfil neuropsicológico y sintomatología psicopatológica de pacientes con trastornos relacionados con sustancias a tratamiento en una unidad de día. Health and Addictions/Salud y Drogas, 19(1), 70-79. https://doi.org/10.21134/haaj.v19i1.412
Rodríguez-Fernández, A., Ramos-Díaz, E., Madariaga, J. M., Arrivillaga, A., \& Galende, N. (2016). Steps in the construction and verification of an explanatory model of psychosocial adjustment. European Journal of Education and Psychology, 9(1), 20-28. $\quad$ https://doi.org/10.1016/j.ejeps.2015.11.002

Ruiz, J., \& Pérez, E. (2014). Neuropsicología de la adicción (1.a ed.). Editorial Médica Panamericana

Salcedo Palacios, D., Ramírez Nova, Y., \& Acosta Barreto, M. (2015). Función y conducta ejecutiva en universitarios consumidores de alcohol. Revista Colombiana de Psiquiatría, 44(1), 3-12. https://doi.org/10.1016/j.rcp.2015.01.004

Simón Saiz, M. J., Fuentes Chacón, R. M., Garrido Abejar, M., Serrano Parra, M. D., Díaz Valentín, M. J., \& Yubero, S. (2020). Perfil de consumo de drogas en adolescentes. Factores protectores. Semergen, 46(1), 33-40. https://doi.org/10.1016/j.semerg.2019.06.001

Stelzer, F., Cervigni, M. A., \& Martino, P. (2010). Bases neurales del desarrollo de las funciones ejecutivas durante la infancia y adolescencia. Revista Chilena de Neuropsicología, 5(3), 176-184. https://dialnet.unirioja.es/servlet/articulo?codigo $=5751571$

Tamayo Lopera, D. A., Merchán Morales, V., Hernández Calle, J. A., Ramírez Brand, S. M., \& Gallo Restrepo, N. E. (2018). Nivel de desarrollo de las funciones ejecutivas en estudiantes adolescentes de los colegios públicos de Envigado-Colombia. CES Psicología, 11(2), 21-36. https://doi.org/10.21615/cesp.11.2.3

Tarter, R. E., Vanyukov, M., Kirisci, L., Reynolds, M., \& Clark, D. B. (2006). Predictors of marijuana use in adolescents before and after licit drug use: examination of the gateway hypothesis. The American journal of psychiatry, 163(12), 2134-2140. jp.2006.163.12.2134 
Tirapu Ustárroz, J., García Molina, A., Ríos Lago, M. \& Ardila Ardila, A. (2012). Neuropsicología de la corteza prefrontal y las funciones ejecutivas [Libro electrónico]. Viguera.https://www.researchgate.net/publication/266563587_Neuropsicologia_de_la_corteza_prefrontal_y_la s_funciones_ejecutivas

Torres, Y., Posada, J., Bareño, J., \& Berbesí, D. (2010). Trastornos por abuso y dependencia de sustancias en población colombiana: su prevalencia y comorbilidad con otros trastornos mentales seleccionados. Revista Colombiana de Psiquiatría, 39, 14-35. https://doi.org/10.1016/s0034-7450(14)60265-1

Vázquez Fernández, M. E., Muñoz Moreno, M. F., Fierro Urturi, A., Alfaro González, M., Rodríguez Carbajo, M. L., Rodríguez Molinero, L. (2014). Consumo de sustancias adictivas en los adolescentes de 13 a 18 años y otras conductas de riesgo relacionadas. Pediatría Atención Primaria, 16 ( 62 ) , $125-134$. h tt p s: / / d o i . or $\mathrm{g} / 10.4321 / \mathrm{s} 1139-76322014000300005$

Verdejo, A., Orozco Giménez, C., Meersmans Sánchez, M., Aguilar de Arcos, F., \& Pérez García, M. (2004). Impacto de la gravedad del consumo de drogas de abuso sobre distintos componentes de la función ejecutiva. Rev Neurol, 38(12), 1109-1116.

https://doi.org/10.33588/r-

n.3812.2003592

Vinaccia, S., \& Quiceno, J. (2012). Calidad de vida relacionada con la salud y enfermedad crónica: estudios colombianos. Psychologia, 6(1), 123-136. https://doi.or$\mathrm{g} / 10.21500 / 19002386.1175$

Weinberg, N. Z., Rahdert, E., Colliver, J. D., \& Glantz, M. D. (1998). Adolescent substance abuse: a review of the past 10 years. Journal of the American Academy of Child and Adolescent Psychiatry, 37(3), 252-261. https://doi.org/10.1097/00004583-199803000-00009
Wongtongkam, N. Ward, P. R., Day, A., \& Winefield, A. H. (2014). The influence of protective and risk factors in individual, peer and school domains on Thai adolescents' alcohol and illicit drug use: a survey. Addictive behaviors, 39(10), 1447-1451. https://doi.org/10.1016/j.addbeh.2014.05.026

Yen, C. N., Wang, C. S., Wang, T. Y., Chen, H. F., \& Chang, H. C. (2011). Quality of life and its correlates among heroin users in Taiwan. The Kaohsiung journal of medical sciences, 27(5), 177-183. jms.2010.09.003

\section{https://doi.org/10.1016/j.k-}

